\title{
ON THE BASICITY OF EIGENFUNCTIONS OF A SECOND-ORDER DIFFERENTIAL OPERATOR WITH A DISCONTINUITY POINT IN WEIGHTED LEBESGUE SPACES
}

\author{
TELMAN B. GASYMOV, AZAMAT M. AKHTYAMOV, AND NIGAR R. AHMEDZADE
}

\begin{abstract}
We propose a new method for proving that eigenfunctions of non-self-adjoint differential operators in weighted Lebesgue spaces form a basis. A spectral problem for a second-order discontinuous differential operator on a finite interval of the real axis with one discontinuity point, which divides the interval into commensurable parts, is considered. The spectral parameter linearly enters both the equation and the transmission condition. Such problems arise when studying the problem of oscillation of a loaded string with fixed ends by the method of separation of variables. A theorem on the basis properties of eigen and associated functions of the spectral problem in weighted spaces $L_{p, \rho} \oplus C$ and $L_{p, \rho}$ with a power weight function $\rho(\cdot)$ satisfying the Muckenhoupt condition is proved.
\end{abstract}

\section{Introduction}

Consider the following spectral problem with a discontinuity point

$$
\left.\begin{array}{rl}
y^{\prime \prime} & (x)+\lambda y(x)=0, x \in\left(0, \frac{1}{3}\right) \cup\left(\frac{1}{3}, 1\right), \\
& y(0)=y(1)=0, \\
& y\left(\frac{1}{3}-0\right)=y\left(\frac{1}{3}+0\right), \\
& y^{\prime}\left(\frac{1}{3}-0\right)-y^{\prime}\left(\frac{1}{3}+0\right)=\lambda M y\left(\frac{1}{3}\right),
\end{array}\right\},
$$

which arise when solving the problem of vibrations of a loaded string with fixed ends. These problems are well illuminated in well-known monographs [3, 35, 15]. Boundary problems with discontinuity condition in the interior point of the interval are often encountered in various fields of mathematics, mechanics, physics, geophysics, and natural sciences. Such problems are usually associated with discontinuous and non-smooth properties of the environment. For example, discontinuous problems arise in electronics when determining parameters of transmission lines with desired technical characteristics [25, 27]. Discontinuous problems also arise when studying transmission properties of one - dimensional discontinuous environments $[23,34]$. Boundary problems with discontinuity condition in

2010 Mathematics Subject Classification. 34L10, 34L20.

Key words and phrases. Eigenfunctions, basicity, discontinuous differential operator, weighted Lebesgue space, Muckenhoupt condition. 
the interior point of the interval arise also in the geophysical models of Earth structure $[1,24]$. For the problem (1.1), (1.2) the completeness of the eigenfunctions is proved in the spaces $L_{p}(0,1) \oplus C$ and $L_{p}(0,1)$ in [19]. In [20], the basicity of a system of eigenfunctions of problem (1.1), (1.2) is proved in Morrey type spaces. In proofs, the authors of [20] used the classical Hausdorff-Young theorem with respect to the systems of sines and cosines, which generally does not hold in weighted spaces, and this creates an additional difficulty in the study of such problems in the weighted case. It should be noted that these spectral problems are very different from the usual ones. The study of the basis properties of systems from the eigenfunctions of spectral problems with a discontinuity point sometimes requires the use of other methods than previously known. In $[8,9]$, a new method for investigating the basis properties of discontinuous differential operators in the spaces $L_{p}(0,1) \oplus C$ and $L_{p}(0,1)$ is proposed. But due to the needs of applications sometimes basis properties of systems consisting of eigen and associated functions of ordinary differential operators in weighted Lebesgue spaces are required. In the case of a simple differentiation operator $y^{\prime}$ or $y^{\prime \prime}$, whose eigenfunctions are the classical system of exponents $\left\{e^{i n x}\right\}_{n=-\infty}^{+\infty}$ or trigonometric systems $\{\sin n x\}_{n=1}^{\infty},\{\cos n x\}_{n=0}^{\infty}$, respectively, with appropriate boundary conditions, the basicity problems in weighted Lebesgue spaces have been sufficiently well studied (see [21, 14, 28, 29, 30, 33, 32, 31]). In [21] it was proved that the Muckenhoupt condition on the weight function is a necessary and sufficient condition for the basicity of systems in weighted Lebesgue spaces. More general results in this direction were obtained in $[12,13]$, where necessary and sufficient conditions for the basicity of the perturbed system of exponents, as well as systems of sines and cosines in weighted Lebesgue spaces, were obtained. However, as we know (or as it seems to us), in the case of ordinary differential operators with general regular two-point, multipoint or discontinuous boundary conditions, the basicity problems of eigen and associated functions in weighted Lebesgue spaces have not been studied (with the exception of self-adjoint case in the weighted Lebesgue space $L_{2, \rho}$ ). In the present paper, improving the methods of $[8,9]$, the condition for the basicity of the eigenfunctions of the spectral problem (1.1), (1.2) is found in weighted Lebesgue spaces with power weight. It should be noted that the basis property of the eigenfunctions of the spectral problem (1.1), (1.2) in non-weighted Lebesgue spaces, as well as in Morrey-type spaces, was studied in $[20,10]$.

\section{Necessary information}

Let us give some results from [19], which we will need throughout the paper.

Theorem 2.1. [19] The spectral problem (1.1), (1.2) has two series of eigenvalues: $\lambda_{1, n}=\left(\rho_{1, n}\right)^{2}, n=1,2, \ldots, \lambda_{2, n}=\left(\rho_{2, n}\right)^{2}, n=0,1,2, \ldots$, where

$$
\left.\begin{array}{l}
\rho_{1, n}=3 \pi n, \\
\rho_{2, n}=\frac{3 \pi n}{2}+\frac{2+(-1)^{n}}{\pi M n}+O\left(\frac{1}{n^{2}}\right) .
\end{array}\right\}
$$

The corresponding eigenfunctions are given by the following expressions

$$
y_{1, n}(x)=\sin 3 \pi n x, x \in[0,1], \quad n=1,2, \ldots,
$$




$$
y_{2, n}(x)=\left\{\begin{array}{l}
\sin \rho_{2, n}\left(x-\frac{1}{3}\right)+\sin \rho_{2, n}\left(x+\frac{1}{3}\right), x \in\left[0, \frac{1}{3}\right], \\
\sin \rho_{2, n}(1-x), x \in\left[\frac{1}{3}, 1\right], \quad n=0,1,2, \ldots .
\end{array} .\right.
$$

Let us construct the linearizing operator for the problem (1.1), (1.2). Denote by $W_{p}^{2}\left(0, \frac{1}{3}\right) \oplus W_{p}^{2}\left(\frac{1}{3}, 1\right)$ the space of functions whose restrictions to intervals $\left(0, \frac{1}{3}\right)$ and $\left(\frac{1}{3}, 1\right)$ belong to Sobolev spaces $W_{p}^{2}\left(0, \frac{1}{3}\right)$ and $W_{p}^{2}\left(\frac{1}{3}, 1\right)$, respectively, where $1<p<\infty$. Let us define the operator $L$ in the following way. As the domain $D(L)$ we take the manifold

$$
\begin{array}{r}
D(L)=\left\{\hat{y}=\left(y(x), M y\left(\frac{1}{3}\right)\right): y(x) \in W_{p}^{2}\left(0, \frac{1}{3}\right) \oplus W_{p}^{2}\left(\frac{1}{3}, 1\right),\right. \\
\left.y(0)=y(1)=0, \quad y\left(\frac{1}{3}-0\right)=y\left(\frac{1}{3}+0\right)\right\},
\end{array}
$$

and for $\hat{y} \in D(L)$ the operator $L$ is defined by the relation

$$
L \hat{y}=\left(-y^{\prime \prime} ; y^{\prime}\left(\frac{1}{3}-0\right)-y^{\prime}\left(\frac{1}{3}+0\right)\right) .
$$

The following lemma is true.

Lemma 2.1. The operator $L$ defined by expressions (2.4), (2.5), is a densely defined closed operator in $L_{p}(0,1) \oplus C$ with compact resolvent. The eigenvalues of the operator $L$ and of the problem (1.1),(1.2) coincide. If $y(x)$ is the eigenfunction (associated function) of problem (1.1), (1.2), then $\hat{y}=\left(y(x) ; M y\left(\frac{1}{3}\right)\right)$ is the eigenvector (associated vector) of the operator $L$.

Proof. To prove the first part of the lemma, we take $\hat{u}=(u, \alpha) \in L_{p}(0,1) \oplus C$ and define the functional $F(\hat{u})$ as follows

$$
F(\hat{u})=M u\left(\frac{1}{3}\right)-\alpha .
$$

Let us also define the following functionals

$$
U_{\nu}(\hat{u})=U_{\nu}(u), \quad \nu=1,2,3,
$$

where

$$
U_{1}(u)=u(0), \quad U_{2}(u)=u(1), \quad U_{3}(u)=u\left(\frac{1}{3}-0\right)-u\left(\frac{1}{3}+0\right) .
$$

Then all the functionals $F, U_{\nu}, \nu=1,2,3$, are linear and bounded in $W_{p}^{2}\left(0, \frac{1}{3}\right) \oplus$ $W_{p}^{2}\left(\frac{1}{3}, 1\right) \oplus C$, but unbounded in $L_{p}(0,1) \oplus C$. Therefore, (see e.g. [26, pp. 2729]), the manifold

$$
\begin{gathered}
D(L)=\left\{\hat{u}=(u(x), \alpha): u(x) \in W_{p}^{2}\left(0, \frac{1}{3}\right) \oplus W_{p}^{2}\left(\frac{1}{3}, 1\right),\right. \\
\left.F(\hat{u})=0, U_{\nu}(\hat{u})=0, \nu=1,2,3\right\}
\end{gathered}
$$

is everywhere dense in $L_{p}(0,1) \oplus C$, and the operator $L$, as a finite-dimensional restriction of the corresponding maximal operator, is a closed operator with a compact resolvent (see e.g. [22, p. 238]). The second part of the lemma is verified directly. Lemma is proved.

When obtaining the main results, we need some concepts and facts from the theory of bases in a Banach space. 
Definition 2.1. Let $X$ be a Banach space. If there exists a sequence of positive integers $\left\{n_{k}\right\}$, such that $n_{k}<n_{k+1}, n_{0}=0$ and any vector $x \in X$ is uniquely represented in the form

$$
x=\sum_{k=0}^{\infty} \sum_{i=n_{k}+1}^{n_{k+1}} c_{i} u_{i},
$$

then the system $\left\{u_{n}\right\}_{n \in N} \in X$ is called a basis with parentheses in $X$. For $n_{k}=k$ the system $\left\{u_{n}\right\}_{n \in N}$ forms an ordinary basis for $X$.

We need the following easily proved statement.

Statement 2.1. Let the system $\left\{u_{n}\right\}_{n \in N}$ form a basis with parentheses for a Banach space $X$. If the sequence $\left\{n_{k+1}-n_{k}\right\}_{k \in N}$ is bounded and the condition

$$
\sup _{n}\left\|u_{n}\right\|\left\|\vartheta_{n}\right\|<\infty
$$

holds, where $\left\{\vartheta_{n}\right\}_{n \in N}$ is a biothogonal system, then the system forms an ordinary basis for $X$.

Recall the following definition.

Definition 2.2. The bases $\left\{u_{n}\right\}_{n \in N}$ of Banach space $X$ is called a $p$-basis, if for any $x \in X$ the condition

$$
\left(\sum_{n=1}^{\infty}\left|\left\langle x, \vartheta_{n}\right\rangle\right|^{p}\right)^{\frac{1}{p}} \leq C\|x\|,
$$

holds, here $\left\{\vartheta_{n}\right\}_{n \in N}$ is a biothogonal system to $\left\{u_{n}\right\}_{n \in N}$.

Definition 2.3. The sequences $\left\{u_{n}\right\}_{n \in N}$ and $\left\{\varphi_{n}\right\}_{n \in N}$ of Banach space $X$ is called a $p$-close, if

$$
\sum_{n=1}^{\infty}\left\|u_{n}-\varphi_{n}\right\|^{p}<\infty .
$$

We will also use the following results from $[6,17,18]$.

Theorem 2.2. Let $\left\{x_{n}\right\}_{n \in N}$ form a q-basis for the space $X$, and the system $\left\{y_{n}\right\}_{n \in N}$ is p-close to $\left\{x_{n}\right\}_{n \in N}$, where $\frac{1}{p}+\frac{1}{q}=1$. Then the following properties of the system are equivalent:

i) $\left\{y_{n}\right\}_{n \in N}$ is complete in $X$;

ii) $\left\{y_{n}\right\}_{n \in N}$ is minimal in $X$;

iii) $\left\{y_{n}\right\}_{n \in N}$ forms an isomorphic basis to $\left\{x_{n}\right\}_{n \in N}$ for $X$.

Let $X_{1}=X \oplus C^{m}$ and $\left\{\hat{u}_{n}\right\}_{n \in N} \subset X_{1}$ be some minimal system, and $\left\{\hat{\vartheta}_{n}\right\}_{n \in N} \subset$ $X_{1}^{*}=X^{*} \oplus C^{m}$ be its biorthogonal system:

$$
\hat{u}_{n}=\left(u_{n} ; \alpha_{n 1}, \ldots, \alpha_{n m}\right) ; \quad \hat{\vartheta}_{n}=\left(\vartheta_{n} ; \beta_{n 1}, \ldots, \beta_{n m}\right) .
$$

Let $J=\left\{n_{1}, \ldots, n_{m}\right\}$ be some set of $m$ natural numbers. Suppose

$$
\delta=\operatorname{det}\left\|\beta_{n_{i} j}\right\|_{i, j=\overline{1, m}} .
$$

The following theorem is true. 
Theorem 2.3. $[17,18]$. Let the system $\left\{\hat{u}_{n}\right\}_{n \in N}$ form a basis for $X_{1}$. In order to the system $\left\{u_{n}\right\}_{n \in N_{J}}$, where $N_{J}=N \backslash J$, form a basis for $X$, it is necessary and sufficient that the condition $\delta \neq 0$ be satisfied. In this case the biorthogonal system to $\left\{u_{n}\right\}_{n \in N_{J}}$ is defined by

$$
\vartheta_{n}^{*}=\frac{1}{\delta}\left|\begin{array}{cccc}
\vartheta_{n} & \vartheta_{n 1} & \ldots & \vartheta_{n m} \\
\beta_{n 1} & \beta_{n_{1} 1} & \ldots & \beta_{n_{m} 1} \\
\ldots & \ldots & \ldots & \ldots \\
\beta_{n m} & \beta_{n_{1} m} & \ldots & \beta_{n_{m} m}
\end{array}\right| .
$$

For $\delta=0$ the system $\left\{u_{n}\right\}_{n \in N_{J}}$ is not complete and is not minimal in $X$.

More details on these and other results can be found in $[6,17,18,11,7]$.

Let $X$ be a Banach space and $\left\{u_{k n}\right\}_{k=\overline{1, m} ; n \in N}$ be some system in $X$. Let $a_{i k}^{(n)}, i, k=\overline{1, m}, n \in N$, be some complex number. Let

$$
A_{n}=\left(a_{i k}^{(n)}\right)_{i, k=\overline{1, m}} \quad \text { and } \quad \Delta_{n}=\operatorname{det} A_{n}, \quad n \in N .
$$

Let us consider the following system in space $X$

$$
\hat{u}_{k n}=\sum_{i=1}^{m} a_{i k}^{(n)} u_{i n}, \quad k=\overline{1, m} ; n \in N .
$$

Theorem 2.4. [10] If the system $\left\{u_{k n}\right\}_{k=\overline{1, m} ; n \in N}$ forms a basis for $X$ and

$$
\Delta_{n} \neq 0, \forall n \in N \text {, }
$$

then the system $\left\{\hat{u}_{k n}\right\}_{k=\overline{1, m} ; n \in N}$ forms a basis with parentheses for $X$. If in addition the conditions

$$
\sup _{n}\left\{\left\|A_{n}\right\|,\left\|A_{n}^{-1}\right\|\right\}<\infty, \quad \sup _{n}\left\{\left\|u_{k n}\right\|,\left\|\vartheta_{k n}\right\|\right\}<\infty,
$$

holds, where $\left\{\vartheta_{k n}\right\}_{k=\overline{1, m} ; n \in N} \subset X^{*}$ is a biorthogonal system to $\left\{u_{k n}\right\}_{k=\overline{1, m} ; n \in N}$, then the system $\left\{\hat{u}_{k n}\right\}_{k=\overline{1, m} ; n \in N}$ forms a usual basis for $X$.

We also need some statements concerning the basicity of trigonometric systems in weighted Lebesgue spaces. Let

$$
\rho(t)=\prod_{k=1}^{m}\left|t-t_{k}\right|^{\alpha_{k}},\left\{t_{k}\right\}_{1}^{m} \subset[0,1], t_{i} \neq t_{j}, i \neq j,
$$

be a weight function. Consider a weighted space $L_{p, \rho}(0,1)$ with a norm

$$
\|f\|_{p, \rho}=\left(\int_{0}^{1}|f(t) \rho(t)|^{p} d t\right)^{\frac{1}{p}} .
$$

The following statement is true.

Statement 2.2. Let the weight $\rho(\cdot)$ satisfy the condition (Muckenhoupt condition)

$$
-\frac{1}{p}<\alpha_{k}<\frac{1}{q}, k=\overline{1, m},
$$

where $\frac{1}{p}+\frac{1}{q}=1$. Then the system of sines $\{\sin \pi n t\}_{n \in N}$ and $\operatorname{cosines}\{\cos \pi n t\}_{n \in Z_{+}}$ form a basis for $L_{p, \rho}(0,1), 1<p<+\infty$. 
This statement follows directly from the corresponding results concerning the system of exponents (see e.g. [21])

Let the condition $(2.7)$ hold and $p_{1} \in(1,+\infty)-$ be some number and $\frac{1}{p_{1}}+\frac{1}{p_{1}^{\prime}}=$ 1. We have

$$
\int_{0}^{1}|f|^{r} d t=\int_{0}^{1}|f \rho|^{r} \rho^{-r} d t \leq\left(\int_{0}^{1}|f \rho|^{r p_{1}} d t\right)^{\frac{1}{p_{1}}}\left(\int_{0}^{1} \rho^{-r p_{1}^{\prime}} d t\right)^{\frac{1}{p_{1}^{\prime}}}
$$

Take

$$
p_{1}=\frac{p}{r}, 1<r<p \Rightarrow \frac{1}{p_{1}^{\prime}}=1-\frac{1}{p_{1}}=1-\frac{r}{p}=\frac{p-r}{p} \Rightarrow p_{1}^{\prime}=\frac{p}{p-r} .
$$

Consequently

$$
\int_{0}^{1}|f|^{r} d t \leq\left(\int_{0}^{1}|f \rho|^{p} d t\right)^{\frac{r}{p}}(I(\rho))^{\frac{1}{p_{1}^{\prime}}}
$$

where

$$
I(\rho)=\int_{0}^{1} \rho^{-\frac{r p}{p-r}} d t .
$$

Thus, it is clear that if $I(\rho)<+\infty$, then $f \in L_{r}(0,1)$. So, if

$$
-\frac{r p}{p-r} \alpha_{k}>-1, k=\overline{1, m}
$$

Then $I(\rho)<+\infty$. We have

$$
\alpha_{k}<\frac{p-r}{r p}=\frac{1}{r}-\frac{1}{p}<\frac{1}{q} .
$$

Since, $\frac{1}{r}-\frac{1}{p} \rightarrow \frac{1}{q}$, as $r \rightarrow 1+0$, then it follows from the conditions (2.7) that $\exists r_{0} \in(1,+\infty)$ :

$$
\alpha_{k}<\frac{1}{r}-\frac{1}{p}, k=\overline{1, m}, \forall r \in\left(1, r_{0}\right) .
$$

So, we have

Statement 2.3. Suppose that conditions (2.7) hold. Then $\exists r_{0} \in(1,+\infty): L_{p, \rho}(0,1) \subset$ $L_{r}(0,1), \forall r \in\left(1, r_{0}\right)$.

Let $f \in L_{p, \rho}(0,1), 1<p<+\infty$, and $\rho(\cdot)$ satisfy the condition (2.7). Then, as it follows from the Statement 2.3, $\exists r \in(1,2): f \in L_{r}(0,1)$. From the HausdorffYoung theorem

$$
\left(\sum\left|f_{n}\right|^{r^{\prime}}\right)^{\frac{1}{r^{\prime}}} \leq C\|f\|_{r} \leq C\|f\|_{p, \rho}
$$

where $\frac{1}{r^{\prime}}+\frac{1}{r}=1, C$ is a constant (may be different in different inequalities). As a result, we get that the system of sines (and cosines also) is $r^{\prime}$-Besselian in $L_{p, \rho}(0,1)$. Let the system $\left\{g_{n}\right\}_{n \in N} \subset L_{p, \rho}(0,1)$ be $r$-close to the system $\left\{f_{n}\right\}$, i.e.

$$
\sum\left\|f_{n}-g_{n}\right\|_{p, \rho}^{r}<+\infty,
$$

where $f_{n}(t)=\sin \pi n t$ (or $\left.f_{n}(t)=\cos \pi n t\right)$. Then it follows from Theorem 2.2 that the following statement holds. 
Statement 2.4. Suppose that for $r \in(1,2)$ there is a continuous embedding $L_{p, \rho}(0,1) \subset L_{r}(0,1)$ and the system $\left\{g_{n}\right\}$ is $r$-close to the system $\left\{f_{n}\right\}$ in $L_{p, \rho}(0,1)$ (where $f_{n}(t)=\sin \pi n t$, or $f_{n}(t)=\cos \pi n t$ ). Then the following properties of the system $\left\{g_{n}\right\}$ are equivalent in $L_{p, \rho}(0,1)$ :

1) $\left\{g_{n}\right\}$ is complete;

2) $\left\{g_{n}\right\}$ is minimal;

3) $\left\{g_{n}\right\}$ forms an isomorphic basis to $\left\{f_{n}\right\}$.

\section{Main results}

In this section we consider the question of the basicity of the system of eigenvectors of the operator $L$ and eigenfunctions of the problem (1.1)-(1.2) in the spaces $L_{p, \rho}(0,1) \oplus C$ and $L_{p, \rho}(0,1)$. The following theorem holds

Theorem 3.1. Let the weight function $\rho(x)$ defined by (2.6) satisfy condition (2.7). Then the system of eigen and associated vectors of the operator $L$ forms a bases for space $L_{p, \rho}(0,1) \oplus C, 1<p<\infty$.

Proof. According to Lemma 2.1 the operator $L$ which linearized the problem (1.1)-(1.2) in the space $L_{p}(0,1) \oplus C$ is densely defined operator with compact resolvent. The system $\left\{\hat{u}_{\mathrm{n}}\right\}_{\mathrm{n}=0}^{\infty}$ of eigen and associated vectors of operator $L$ is minimal in the space $L_{p}(0,1) \oplus C$ (see [2, p.40], [16, pp. 380-385, 401-402]) and its conjugate system $\left\{\hat{z}_{n}\right\}_{n=0}^{\infty}$ is the system of eigenvectors of the adjoint operator $L^{*}$ and it is of the form

$$
\hat{z}_{n}=\left(z_{n}, \bar{M} z_{n}\left(\frac{1}{3}\right)\right), n=0,1, \ldots,
$$

Here $z_{n}(x), n=0,1, \ldots$, are the eigenfunctions of adjoint spectral problem

$$
\begin{gathered}
z^{\prime \prime}(x)+\lambda z(x)=0, \quad x \in\left(0, \frac{1}{3}\right) \cup\left(\frac{1}{3}, 1\right), \\
z(0)=z(1)=0 ; z\left(\frac{1}{3}-0\right)=z\left(\frac{1}{3}+0\right) ; \\
z^{\prime}\left(\frac{1}{3}-0\right)-z^{\prime}\left(\frac{1}{3}+0\right)=\lambda \bar{M} z\left(\frac{1}{3}\right) .
\end{gathered}
$$

Carrying out similar arguments for the problem (3.2), (3.3) we obtain that for $\vartheta_{n}(x), n=0,1, .$. , the following formulas are valid

$$
\begin{gathered}
z_{1, n}(x)=2 \sin 3 \pi n x, \quad x \in[0,1], \quad n=1,2, \ldots, \\
z_{2, n}(x)=\left\{\begin{array}{l}
c_{2, n}\left(\sin \frac{3 \pi n}{2}\left(x-\frac{1}{3}\right)+\sin \frac{3 \pi n}{2}\left(x+\frac{1}{3}\right)\right)+O\left(\frac{1}{n}\right), \quad x \in\left[0, \frac{1}{3}\right], \\
c_{2, n} \sin \frac{3 \pi n}{2}(1-x)+O\left(\frac{1}{n}\right), \quad x \in\left[\frac{1}{3}, 1\right], \quad n=0,1,2, \ldots,
\end{array}\right.
\end{gathered}
$$

where $c_{2, n}$ are the normalization numbers and for which the asymptotic relation

$$
c_{2, n}=\frac{2+(-1)^{n}}{3}+O\left(\frac{1}{n}\right)
$$


holds. Denote

$$
\begin{aligned}
& u_{1, n}(x)=\sin 3 \pi n x, \quad x \in[0,1], \quad n=1,2, \ldots, \\
& u_{2, n}(x)=\left\{\begin{array}{l}
2(-1)^{n} \sin 3 \pi n x, \quad x \in\left[0, \frac{1}{3}\right], \\
\sin 3 \pi n x, \quad x \in\left[\frac{1}{3}, 1\right],
\end{array}\right. \\
& u_{3, n}(x)=\left\{\begin{array}{l}
0, \quad x \in\left[0, \frac{1}{3}\right], \\
-\cos 3 \pi\left(n-\frac{1}{2}\right) x, \quad x \in\left[\frac{1}{3}, 1\right] .
\end{array}\right.
\end{aligned}
$$

From the asymptotic formulas (2.1) - (2.3) it follows that

$$
\left.\begin{array}{l}
y_{1, n}(x)=u_{1, n}(x)+O\left(\frac{1}{n}\right), \\
y_{2,2 n}(x)=u_{2, n}(x)+O\left(\frac{1}{n}\right), \\
y_{2,2 n-1}(x)=u_{3, n}(x)+O\left(\frac{1}{n}\right) .
\end{array}\right\}
$$

It is not difficult to see that the system (3.6) is obtained from the system $\left\{e_{i, n}\right\}_{i=\overline{1,3} ; n \in N}$ by means of a transformation carried out by the matrix

$$
A_{n}=\left(\begin{array}{ccc}
1 & 1 & 0 \\
2(-1)^{n} & 1 & 0 \\
0 & 0 & 1
\end{array}\right)
$$

where

$$
\begin{aligned}
& e_{1, n}(x)=\left\{\begin{array}{l}
\sin 3 \pi n x, x \in\left[0, \frac{1}{3}\right], \\
0, \quad x \in\left[\frac{1}{3}, 1\right],
\end{array}\right. \\
& e_{2, n}(x)=\left\{\begin{array}{l}
0, \quad x \in\left[0, \frac{1}{3}\right], \\
\sin 3 \pi n x, \quad x \in\left[\frac{1}{3}, 1\right],
\end{array}\right. \\
& e_{3, n}(x)=\left\{\begin{array}{l}
0, \quad x \in\left[0, \frac{1}{3}\right], \\
-\cos 3 \pi\left(n-\frac{1}{2}\right) x, \quad x \in\left[\frac{1}{3}, 1\right] .
\end{array}\right.
\end{aligned}
$$

Here the transformation is understood in the sense of

$$
u_{i, n}=\sum_{j=1}^{3} a_{i j}^{(n)} e_{j, n},
$$

where $a_{i j}^{(n)}$ are the elements of the matrix $A_{n}$. Note that for every $n \in N$

$$
\operatorname{det} A_{n}=1-2(-1)^{n} \neq 0 .
$$

Consider the system $\left\{\hat{u}_{0}\right\} \cup\left\{\hat{u}_{i, n}\right\}_{i=\overline{1,3} ; n \in N}$ in space $L_{p, \rho}(0,1) \oplus C$, where

$$
\hat{u}_{0}=(0 ; 1), \hat{u}_{i, n}=\left(u_{i, n} ; 0\right), \quad i=\overline{1,3} ; n \in N .
$$

By virtue of the decomposition

$$
L_{p, \rho}(0,1)=L_{p, \rho}\left(0, \frac{1}{3}\right) \oplus L_{p, \rho}\left(\frac{1}{3}, 1\right),
$$

(which is easy to set in a standard way), and also due to the fact that the trigonometric systems $\{\sin 3 \pi n x\}_{n \in N}$ and $\{\sin 3 \pi n x ; \cos 3 \pi(n-1 / 2) x\}_{n \in N}$ are a bases in spaces $L_{p, \rho}(0 ; 1 / 3)$ and $L_{p, \rho}(1 / 3 ; 1)$ consequently (see. the Statement 2.2), Theorem 2.4 implies that the system $\left\{u_{i, n}\right\}_{i=\overline{1,3} ; n \in N}$ forms a bases for $L_{p, \rho}(0,1), 1<p<\infty$. Then it is obvious that the system $\left\{\hat{u}_{0}\right\} \cup\left\{\hat{u}_{i, n}\right\}_{i=1,3 ; n \in N}$ will form a basis for $L_{p, \rho}(0,1) \oplus C$. Let us show that it forms a $r$-basis for this 
space for some $r>1$. It is easy to verify that the system biorthogonal to it $\left\{\hat{\vartheta}_{0}\right\} \cup\left\{\hat{\vartheta}_{i, n}\right\}_{i=\overline{1,3} ; n \in N}$ has the form

$$
\hat{\vartheta}_{0}=(0 ; 1), \quad \hat{\vartheta}_{i, n}=\left(\vartheta_{i, n} ; 0\right), \quad i=\overline{1,3} ; n \in N
$$

where

$$
\begin{aligned}
& \vartheta_{1, n}(x)= \begin{cases}\frac{6}{1-2\left(-1^{n}\right)} \sin 3 \pi n x, & x \in\left[0, \frac{1}{3}\right], \\
-\frac{6(-1)^{n}}{1-2(-1)^{n}} \sin 3 \pi n x, & x \in\left[\frac{1}{3}, 1\right],\end{cases} \\
& \vartheta_{2, n}(x)= \begin{cases}\frac{-6}{1-2\left(-1^{n}\right)} \sin 3 \pi n x, & x \in\left[0, \frac{1}{3}\right], \\
\frac{3}{1-2(-1)^{n}} \sin 3 \pi n x, & x \in\left[\frac{1}{3}, 1\right], \\
0, \quad x \in\left[0, \frac{1}{3}\right], & \\
-3 \cos 3 \pi\left(n-\frac{1}{2}\right) x, & x \in\left[\frac{1}{3}, 1\right] .\end{cases}
\end{aligned}
$$

Let $1<p \leq 2$. Then, by Statement 2.3, there exists $r: 1<r<2$, such that $L_{p, \rho}(0,1) \subset L_{r}(0,1)$. Then, by the Hausdorff-Young inequality, for a trigonometric system (see, for example, [10] ) for all $f \in L_{r}(0,1)$ the inequality

$$
\left(\sum_{i=1}^{3} \sum_{n=1}^{\infty}\left|<f, e_{i, n}>\right|^{r^{\prime}}\right)^{\frac{1}{r^{\prime}}} \leq\|f\|_{L_{r}},
$$

holds, where $\frac{1}{r^{\prime}}+\frac{1}{r}=1$, and $>0$ is some constant independent of $f$. Taking into account that the system $\left\{\vartheta_{i, n}\right\}_{i=\overline{1,3} ; n \in N}$ is obtained from the system $\left\{e_{i, n}\right\}_{i=\overline{1,3} ; n \in N}$ by transforming

$$
u_{i, n}=\sum_{j=1}^{3} b_{i j}^{(n)} e_{j, n},
$$

where $b_{i j}^{(n)}$ are elements of the matrix $\left(A_{n}^{-1}\right)^{*}$ and for which the condition

$$
\sup _{n}\left\{\left\|\left(A_{n}^{-1}\right)^{*}\right\|\right\}<+\infty
$$

holds, we obtain that for all $\hat{f} \in L_{r}(0,1) \oplus C$ the following inequality is also fulfilled

$$
\left(\sum_{i=1}^{3} \sum_{n=1}^{\infty}\left|\left\langle\hat{f}, \hat{\vartheta}_{i, n}\right\rangle\right|^{r^{\prime}}\right)^{\frac{1}{r^{\prime}}} \leq\|\hat{f}\|_{L_{r} \oplus C}
$$

Then taking into account the embedding $L_{p, \rho}(0,1) \subset L_{r}(0,1)$ we obtain that for all $\hat{f} \in L_{p, \rho}(0,1) \oplus C$ the inequality

$$
\left(\sum_{i=1}^{3} \sum_{n=1}^{\infty}\left|\left\langle\hat{f}, \hat{\vartheta}_{i, n}\right\rangle\right|^{r^{\prime}}\right)^{\frac{1}{r^{\prime}}} \leq\|\hat{f}\|_{L_{p, \rho} \oplus C},
$$

holds, i.e. the system $\left\{\hat{u}_{i, n}\right\}_{i=\overline{1,3} ; n \in N}$ forms a $r^{\prime}$-basis for $L_{p, \rho}(0,1) \oplus C$. Let us denote

$$
\begin{gathered}
\hat{y}_{1, n}=\left(y_{1, n}(x) ; M y_{1, n}\left(\frac{1}{3}\right)\right), \\
\hat{y}_{2, n}=\left(y_{2,2 n}(x) ; M y_{2,2 n}\left(\frac{1}{3}\right)\right),
\end{gathered}
$$




$$
\hat{y}_{3, n}=\left(y_{2,2 n-1}(x) ; M y_{2,2 n-1}\left(\frac{1}{3}\right)\right), \quad n \in N .
$$

Then it follows from the asymptotic formulas (3.7) that the systems $\left\{\hat{y}_{i, n}\right\}_{i=\overline{1,3} ; n \in N}$ and $\left\{\hat{u}_{i, n}\right\}_{i=\overline{1,3} ; n \in N}$ are $r$-close, i.e.

$$
\sum_{i=1}^{3} \sum_{n=1}^{\infty}\left\|\hat{y}_{i, n}-\hat{u}_{i, n}\right\|_{L_{p, \rho} \oplus C}^{r}<\infty
$$

On the other hand, by Theorem 2.4 the system $\left\{\hat{u}_{0}\right\} \cup\left\{\hat{u}_{i, n}\right\}_{i=\overline{1,3} ; n \in N}$ forms a bases for the space $L_{r}(0,1) \oplus C$, therefore, it is minimal in this space and in view of the embedding

$$
\left(L_{r^{\prime}}(0,1) \oplus C\right) \subset\left(L_{q, \rho}(0,1) \oplus C\right),
$$

we find that it is minimal in $L_{q, \rho}(0,1) \oplus C$. Thus, all the conditions of Statement 2.4 hold and therefore the system $\left\{\hat{y}_{0}\right\} \cup\left\{\hat{y}_{i, n}\right\}_{i=1,3 ; n \in N}^{\infty}$ forms an equivalent basis to the system $\left\{\hat{u}_{0}\right\} \cup\left\{\hat{u}_{i, n}\right\}_{i=\overline{1,3} ; n \in N}$ for space $L_{p, \rho}(0,1) \oplus C$.

Now, let $p>2$ and $\hat{f} \in L_{p, \rho}(0,1) \oplus C$ be an arbitrary element. Then $\hat{f} \in$ $L_{q, \rho}(0,1) \oplus C$ and by Statement 2.3 there exists $r, 1<r<2$, such that $\hat{f} \in$ $L_{r}(0,1) \oplus C$ and by Hausdorff-Young inequality and by virtue of the embeddings

$$
L_{p, \rho}(0,1) \subset L_{q, \rho}(0,1), l_{r} \subset l_{r^{\prime}},
$$

we obtain the following inequality

$$
\left(\sum_{i=1}^{3} \sum_{n=1}^{\infty}\left|\left\langle\hat{f}, \hat{\vartheta}_{i, n}\right\rangle\right|^{r^{\prime}}\right)^{\frac{1}{r^{\prime}}} \leq\|\hat{f}\|_{L_{r} \oplus C} \leq\|\hat{f}\|_{L_{q, \rho} \oplus C} \leq C\|\hat{f}\|_{L_{p, \rho} \oplus C} .
$$

This means that the system $\left\{\hat{u}_{0}\right\} \cup\left\{\hat{u}_{i, n}\right\}_{i=\overline{1,3} ; n \in N}$ is a $r^{\prime}$-bases in $L_{p, \rho}(0,1) \oplus C$. Moreover

$$
\sum_{i=1}^{3} \sum_{n=1}^{\infty}\left\|\hat{y}_{i, n}-\hat{u}_{i, n}\right\|_{L_{p, \rho}}^{r}<\infty
$$

and the system $\left\{\hat{y}_{0}\right\} \cup\left\{\hat{y}_{i, n}\right\}_{i=\overline{1,3 ; n} \in N}^{\infty}$ is minimal in $L_{p, \rho}(0,1) \oplus C$, then again applying Theorem 2.2, we find that it forms a basis for the space $L_{p, \rho}(0,1) \oplus C$ isomorphic to the system $\left\{\hat{u}_{0}\right\} \cup\left\{\hat{u}_{i, n}\right\}_{i=\overline{1,3} ; n \in N}$. This completes the proof.

Now, let us consider the basicity of the system $\left\{y_{0}\right\} \cup\left\{y_{i, n}\right\}_{i=1,2 ; n \in N}^{\infty}$ with a remote function in space $L_{p, \rho}(0,1)$.

Theorem 3.2. If from the system of eigen and associated functions of problem (1.1)-(1.2) $\left\{y_{0}\right\} \cup\left\{y_{i, n}\right\}_{i=1,2 ; n \in N}^{\infty}$ we eliminate any function $y_{2, n_{0}}(x)$, corresponding to a simple eigenvalue, then the obtaining system forms a basis for $L_{p, \rho}(0,1)$, $1<p<\infty$. And if we eliminate any function $y_{1, n_{0}}(x)$ from this system, then the obtaining system does not form a basis in $L_{p, \rho}(0,1)$; moreover, in this case the obtained system is not complete and is not minimal in this space.

Proof. From formulas (3.4), (3.5) for the eigenfunctions $\left\{z_{0}\right\} \cup$ $\left\{z_{i, n}\right\}_{i=1,2 ; n \in N}^{\infty}$ of the adjoint problem it follows that $z_{1, n}\left(\frac{1}{3}\right)=0$ for any $n \in N$ and $z_{2, n}\left(\frac{1}{3}\right) \neq 0$ for any eigenfunction corresponding to a simple eigenvalue $\bar{\lambda}_{2, n}=$ $\bar{\rho}_{2, n}^{2}$. On the other hand, the eigenvectors of the adjoint operator are defined by 
(3.1). Applying Theorem 2.2 to the system, we notice that $\delta=\bar{M} z_{1, n}\left(\frac{1}{3}\right)=0$ for any $n \in N$ and $\delta=\bar{M} z_{2, n}\left(\frac{1}{3}\right) \neq 0$ for any eigenfunction corresponding to a simple eigenvalue, and the statements of the theorem follow from the corresponding statements of Theorem 2.3.

Remark 3.1. For $M>0$, the linearizing operator $L$ of the problem (1.1)-(1.2) is a self-adjoint operator in $L_{2} \oplus C$ and in this case all eigenvalues are real and simple, and to each eigenvalue there corresponds only one eigenvector. If $M<0$, then the operator $L$ is $J$ self-adjoint operator in $L_{2} \oplus C$ and hence, applying the results of $[4,5]$, we get that either (i) all eigenvalues of the problem are simple; in that case it may have just two non-real, mutually conjugate eigenvalues; or (ii) all eigenvalues of the problem are real; in that case it may have at most one multiple eigenvalue. In the case of a complex value $M$ the operator $L$ has an infinite number of complex eigenvalues that are asymptotically simple and, consequently, the operator $L$ can have a finite number of associated vectors. If there are associated vectors, they are determined up to a linear combination with the corresponding eigenvector, and in this case there always exists an associated vector for which $z_{2, n}\left(\frac{1}{3}\right)=0$, as well as an associated vector for which $z_{2, n}\left(\frac{1}{3}\right) \neq 0$.

\section{Acknowledgements}

This work was supported by the Science Development Foundation under the President of the Republic of Azerbaijan - Grant No. EIF-BGM-4-RFTF-1/201721/02/1-M-19

\section{References}

[1] R.S. Anderssen, The effect of discontinuities in density and shear velocity on the asymptotic overtone structure of torsional eigenfrequencies of the Earth, Geophys. J. R. Astr. Soc., 50 ( 1997), 303-309.

[2] M.S. Agranovich, Elliptic operators on closed manifolds, In contemporary Problems of Math., Fundamental Directions, 63, 5-129, VINITI, Moscow, 1989 (Russian; Engl. Transl. in Encycl. of Math. Sc., 63, 1-130, Springer-Verlag, 1994 )

[3] F.V. Atkinson, Discrete and Continuous Boundary Problems, Moscow, Mir, 1968.

[4] T.Ya. Azizov, I.S. Iokhvidov, A criterion for the completeness and basicity of root vectors of a completely continuous $J$-selfadjoint operator in the Pontryagin space $\Pi_{æ}$, Math. iss., 6(1) (1971), 158-161. (in Russian)

[5] T.Ya. Azizov, I.S. Iokhvidov, Linear operators in Hilbert spaces with a $G$-metric, Uspekhi. mat. Science, 26(4) (1971), 43-92. (in Russian)

[6] B.T. Bilalov, Bases of Exponentials, Sines, and Cosines, Differ. Uravn., 39(5) (2003), 619-623.

[7] B.T. Bilalov, Some problems of approximation, Baku, Elm, 2016, 380 p. (in Russian)

[8] B.T. Bilalov, T.B. Gasymov, On bases for direct decomposition, Doklady Mathematics, 93(2) (2016), 183-185.

[9] B.T. Bilalov, T.B. Gasymov, On basicity a system of eigenfunctions of second order discontinuous differential operator, Ufa Mathematical Journal,9(1) (2017), 109-122.

[10] B.T. Bilalov, T.B. Gasymov, G.V. Maharramova, On basicity of eigenfunctions of one discontinuous spectral problem in Morrey type space, EJDE (submitted).

[11] B.T. Bilalov, Z.G. Guseynov, $K$-Bessel and $K$-Hilbert systems and $K$-bases, Doklady Mathematics, 80(3) (2009), 826-828. 
[12] B.T. Bilalov, S.G. Veliev, On completeness of exponent system with complex coefficients in weighted spaces, Trans. Natl. Acad. Sci. Azerb. Ser. Phys.-Tech. Math. Sci., 25(7) (2005), 9-14.

[13] B.T. Bilalov, S.G. Veliev, Bases of eigenfunctions of two discontinuous differential operators, Differ. Equ., 42(10) (2006), 1503-1506.

[14] A. Boivin, A.M. Sedletskii, Bases of exponentiales in weighted $L_{p}$-spaces, Spectral and evolution problems, 14. Proc. 14 Crimean Autumn Math. School - Symp., September 2003, Sevastopol, Laspi, Simferopol, 2004, pp.41-43.

[15] L. Collatz, Eigenvalue Problems, Moscow, Fizmatgiz, 1968, 504 p (in Russian).

[16] N. Dunford, J.T. Schwartz, Linear operators, Vol. 3., Spectral operators, Moscow, Mir, 1971, $661 \mathrm{p}$.

[17] T.B. Gasymov, On necessary and sufficient conditions of basicity of some defective systems in Banach spaces, Trans. NAS Azerb., ser. phys.-tech. math. sci., math.mech., 26(1) (2006), 65-70.

[18] T.B. Gasymov, T.Z. Garayev, On necessary and sufficient conditions for obtaining the bases of Banach spaces, Proc. of IMM of NAS of Azerb., XXVI(XXXIV) (2007), 93-98.

[19] T.B. Gasymov, G.V. Maharramova, On completeness of eigenfunctions of the spectral problem, Caspian Journal of Applied Mathematics, Ecology and Economics, 3(2) (2015), 66-76.

[20] T.B. Gasymov, G.V. Maharramova, N.G. Mammadova, Spectral properties of a problem of vibrations of a loaded string in Lebesgue spaces, Trans. of NAS of Azerb., Ser. Phys.-Tech. Math. Sci., 38(1) (2018), 62-68.

[21] R.A. Hunt, B. Muckenhoupt, R.L. Wheeden, Weighted norm inequalities for the conjugate function and Hilbert transform, Trans. of Amer. Math. Soc., 176 (1973), $227-251$.

[22] T. Kato, Perturbation theory of linear operators, Moscow, Mir, 1972, 740 p.

[23] R.J. Krueger, Inverse problems for nonabsorbing media with discontinuous material properties, J. Math. Phys., 23(3) (1982), 396-404.

[24] F.R. Lapwood, T. Usami, Free oscillations of the Earth, Cambridge: Cambridge University Press, 1981.

[25] O.N. Litvinenko, V.I. Soshnikov, Theory of Nonuniform Lines and Their Use in Radio Engineering, Moscow: Sov. Radio, 1964 (in Russian).

[26] V.E. Lyantse, O.G. Storozh, Methods of the theory of unbounded operators, Naukova Dumka, Kiev, 1983, 212 p. (in Russian)

[27] V.P. Meshchanov, A.L. Feldstein, Automated Design of Microwave Directional Couplers (Svyaz, Moscow, 1980) (in Russian)

[28] E.I. Moiseev, On the basis property of sine and cosine systems, Dokl. Akad. Nauk SSSR, 275(4) (1984), 794-798.

[29] E.I. Moiseev, On the basis property of sine and cosine systems in a weighted space, Differential Equations, 34(1) (1998), 39-43.

[30] E.I. Moiseev, The basicity in the weight space of a system of eigen functions of a differential operator, Diff. Uravn., 35(2) (1999), 200-205.

[31] S.S. Pukhov, Bases of exponentials, sines and cosines in weighted spaces on a finite interval, Izv. RAN. Ser. Mat., 75(2) (2011), 195-224

[32] S.S. Pukhov, A.M. Sedletskii, Bases of exponentials, sines, and cosines in weighted spaces on a finite interval, Doklady Akademii Nauk, 425(4) (2009), 452-455.

[33] A.M. Sedletskii, Classes of analytic Fourier transforms and exponential approximations, 2005 (Fizmatlit, Moscow), Ch. 9 (in Russian) 
[34] D.G. Shepelsky, The inverse problem of reconstruction of the medium's conductivity in a class of discontinuous and increasing functions, Adv. Sov. Math., 19 (1994), 209-231.

[35] A.N. Tikhonov, A.A. Samarskii, Equations of Mathematical Physics (Mosk. Gos. Univ., Moscow (1999); Dover, New York, (2011).

[36] A. Zigmund, Trigonometric series, 2, Moscow, Mir, 1965, 537 p.

Telman B. Gasymov

Department of "Non-harmonic analysis", Institute of Mathematics and Mechanics of NAS of Azerbaijan, AZ1141, Baku, Azerbaijan

Department of "Theory of functions and functional analysis", Baku State University, AZ1148, Baku, Azerbaijan.

E-mail address: telmankasumov@rambler.ru

Azamat M. Akhtyamov

Department Mathematical Modelling, Bashkir State University, Russia.

E-mail address: AkhtyamovAM@mail.ru

Nigar R. Ahmedzade

Department of "Differential equations", Institute of Mathematics and Mechanics of NAS of Azerbaijan, AZ1141, Baku, Azerbaijan.

E-mail address: nigar_sadigova11@mail.ru

Received: August 16, 2019; Revised: November 17, 2019; Accepted: November 26, 2019 